\section{Pulsatile ocular blood flow in patients with Graves' ophthalmopathy}

Chieh-Chih Tsai ${ }^{1,2,3}$, Hui-Chuan $\mathrm{Kau}^{3,4}$, Shu-Ching Kao ${ }^{1,2}$, Ming-Wei Lin ${ }^{3}$, Wen-Ming $\mathrm{Hsu}^{1,2}$, Jorn-Hon $\mathrm{Liu}^{2,5}$ and Yau-Huei Wei ${ }^{6}$

\section{Introduction}

Purpose Graves' ophthalmopathy (GO) is a cosmetically disfiguring and visionthreatening complication of Graves' disease (GD). We investigated the clinical application of pulsatile ocular blood flow (POBF) measurement for detection of clinically active GO.

Methods In this prospective study, the OBF tonometer (OBF Labs, Wiltshire, UK) was used to measure POBF in 83 eyes of 83 patients with GD in two groups and 42 normal control subjects. Group A comprised 35 GD patients without ophthalmopathy, and group B composed 48 GD patients with ophthalmopathy. Results were compared with controls.

Results Pulse amplitude, pulse volume, and POBF were significantly lower in group $B$ than that in group $A$ and controls $(P<0.001)$. After adjusting for age, sex, systolic blood pressure, diastolic blood pressure, heart rate, intraocular pressure, and axial length, multivariate linear regression analysis showed that POBF was still significantly lower in eyes with GO (772.4 (SD 279.3) $\mu \mathrm{l} / \mathrm{min}$ ) than group A (1177.3 (SD 326.3) $\mu \mathrm{l} / \mathrm{min})$ and controls (1255.4 (SD 295.1) $\mu \mathrm{l} / \mathrm{min}$ ) $(P<0.001)$.

Conclusions These data show that the pulsatile ocular blood flow differences between the groups are relevant to the presence of GO. POBF measurement is a clinically useful adjunct for assessing the haemodynamic change in $\mathrm{GO}$ patients.

Eye (2005) 19, 159-162. doi:10.1038/sj.eye.6701434

Published online 18 June 2004

Keywords: Graves' disease; ophthalmopathy; pulsatile
Among the techniques for ocular blood flow study, pulsatile ocular blood flow (POBF) measurement is easily and quickly performed and the reproducibility is relatively acceptable. ${ }^{1-3}$ Langham and co-workers ${ }^{4}$ first adapted the pneumotonometer to measure the pulsatile change of intraocular pressure (IOP) and converted it into a volume pulse to obtain a measure of POBF. The pulsatile component of ocular blood flow is primarily choroidal. ${ }^{5}$ It has been clinically applied to investigate in various ocular diseases including glaucoma, ${ }^{6,7}$ age-related macular degeneration, ${ }^{8,9}$ diabetic retinopathy, ${ }^{10,11}$ choroidal melanoma, ${ }^{12}$ and retinal pigmentosa. $^{13}$

Graves' disease (GD) is an autoimmune disorder characterized by hyperthyroidism, diffuse goitre, ophthalmopathy, and rarely, dermopathy. One hypothesis for the pathogenesis of Graves' ophthalmopathy (GO) is that the immune response to thyroidstimulating hormone (TSH) receptor-like protein in orbital connect tissue initiates cytokine formation, promoting production by orbital fibroblasts of hydrophilic

glycosaminoglycans, resulting in increased osmotic pressure, extraocular muscle volume, fluid accumulation, and clinical ophthalmopathy. ${ }^{14}$ In two studies of colour Doppler imaging, the investigators detected a significant difference in the vascular velocity of GD patients. ${ }^{15,16}$ However, the alterations in pulsatile ocular blood flow have not been documented in GO. The aim of this study was to assess the clinical usefulness of POBF for the detection of the haemodynamic differences in GD patients with or without ophthalmopathy.
${ }^{1}$ Department of Ophthalmology Taipei Veterans General Hospital Taiwan, ROC

${ }^{2}$ School of Medicine National Yang-Ming University, Taiwan ROC

${ }^{3}$ Institute of Clinical Medicine

National Yang-Ming

University

Taiwan, ROC

${ }^{4}$ Department of

Ophthalmology

Taoyuan Veterans

Hospital

Taiwan, ROC

${ }^{5}$ Department of Ophthalmology Cheng Hsin Rehabilitation Medical Center Taiwan, ROC

${ }^{6}$ Department of Biochemistry and Center for Cellular and Molecular Biology National Yang-Ming University, Taiwan ROC

Correspondence: C-C Tsai Department of Ophthalmology Taipei Veterans General Hospital, No. 201 Sec. 2 Shih-Pai Road

Taiwan, Taipei, ROC Tel: + 886228757325 Fax: + 886228213984

E-mail: cctsai@

vghtpe.gov.tw

None of the authors have any commercial interest in the material mentioned herein.

Received: 9 May 2003 Accepted 29 December 2003

Published online: 18 June 2004 


\section{Materials and methods}

\section{Patients and definition}

This is a prospective, comparative study. Study subjects were eligible for inclusion if they had GD examined at Taipei Veterans General Hospital from July 2001 to Mar 2003 and were divided into group A consisting of GD only and group B consisting of GD with ophthalmopathy. The non-GD controls were recruited from age- and sexmatched healthy people enrolled when they attended their routine healthy examination. Only one eye of each subject was included. The study had local committee approval and written informed consent was obtained from each patient after the nature of the study had been explained.

The diagnosis of GD was established by experienced endocrinologists with objective evidence of abnormal thyroid dysfunction (symptoms and signs of hyperthyroidism, elevated serum levels of free thyroxin (T4), and free tri-iodothyronin (T3) with TSH levels below the lower normal limit before initiating ATD treatment. GD patients were divided into two major groups according to the presence of ophthalmopathy. GO was diagnosis by an experienced ophthalmologist, according to the criteria proposed by Bartley and Gorman. ${ }^{17}$ Study subjects were excluded if they had any ocular disease other than GO or with a history of ocular surgery or radiation therapy. All the GD patients had been free of local or systemic medication for the treatment of ophthalmopathy and were maintained in a euthyroid state at least 1 month before and during the study period. Also excluded in three groups were patients with high myopia more than 6 dioptres equivalent sphere, pregnancy, and systemic vascular diseases (such as systemic hypertension and diabetes). Eligible subjects underwent assessment of refraction, best-corrected visual acuity, extraocular movement, axial length, slit lamp-biomicroscopy, and fundus examinations. Coronal computed tomography (CT) of the orbit and Hertel's exophthalmometry was carried out to help to confirm the diagnosis of GO. All subjects had their blood pressure recorded in the sitting position by a sphygmomanometer after a 5-min rest period.

\section{POBF measurements}

One eye of each control subject and of GD patients without ophthalmopathy was randomly selected to measure POBF. In GD patients with ophthalmopathy, if both eyes had ophthalmopathy, selection of the eye to be examined was random, whereas if only one eye had ophthalmopathy the POBF of the affected eye was measured. IOP was measured with a noncontact tonometer (CT-80, Topcon, Japan). All patients were measured for POBF by an experienced examiner who was masked to the diagnosis. POBF, pulse amplitude (PA), and pulse volume (PV) were measured with an OBF Tonometer (OBF Ltd, Wiltshire, UK) using the software version 16.2.

\section{Statistical analysis}

Data are expressed as means and standard deviations. Univariate comparisons of categorical and continuous variables with group were performed using $\chi^{2}$-test and a one-way analysis of variance (ANOVA) followed by $t$-test using the Bonferroni correction, respectively. To adjust the effects of other variables, multivariate linear regression analysis was performed to evaluate the effect of disease status on the POBF. All statistical comparisons were performed using the SPSS 10.0 for Windows (SPSS Inc). Statistical significance was set at $P<0.05$.

\section{Results}

A total of 83 eyes of 83 GD patients and 42 eyes of 42 control subjects were enrolled in this study. Group A comprised of 35 eyes without ophthalmopathy, and group B comprised 48 eyes with ophthalmopathy. Table 1 shows the baseline clinic data of the three groups. No statistically differences in age, sex, axial length, systolic, and diastolic blood pressures, or heart rate were observed between the subjects in the three groups. However, the mean IOP was significant higher in group $\mathrm{B}(17.6 \mathrm{mmHg})$ than in group $\mathrm{A}(16.0 \mathrm{mmHg})$ and normal subjects ( $15.0 \mathrm{mmHg}, P=0.002)$. The mean values of PA, $\mathrm{PV}$, and POBF in the three groups are shown in Table 2. In the patients with GO, the POBF $(772.4 \mu \mathrm{l} / \mathrm{min}), \mathrm{PV}$ $(4.9 \mu \mathrm{l})$ and PA $(2.3 \mathrm{mmHg})$ were significantly lower than in the patients with GD $(1177.3 \mu \mathrm{l} / \mathrm{min}(P<0.001), 7.6 \mu \mathrm{l}$, and $3.2 \mathrm{mmHg}(P<0.001)$, respectively) and control subjects $(1255.4 \mu \mathrm{l} / \mathrm{min}(P<0.001), 8.7 \mu \mathrm{l}$, and $3.5 \mathrm{mmHg}$ $(P<0.001)$, respectively). There were no significant differences in PA, PV, and POBF between group B and the controls ( $P=0.357,0.122$, and 0.766 , respectively). After adjusting for age, sex, systolic, and diastolic blood pressures, heart rate, IOP, and axial length, multivariate linear regression analysis demonstrated that disease status $(\mathrm{GO})(P<0.001)$ and IOP $(P<0.001)$ were significantly correlated with $\mathrm{POBF}$ and there was no interaction effect between GO and IOP $(P=0.189)$.

\section{Discussion}

GO develops before the onset of hyperthyroidism in about $20 \%$, at the same time as hyperthyroidism in about $40 \%$, and after hyperthyroidism in about $40 \% .{ }^{18}$ These different expressions of the same disease are still poorly 
Table 1 Characteristics of controls and patients with GD or GO

\begin{tabular}{|c|c|c|c|c|}
\hline & Controls $(\mathrm{n}=42)$ & $A(G D)(\mathrm{n}=35)$ & $B(G O)(\mathrm{n}=48)$ & $P$ \\
\hline Age (years) & $47.8(18.0)$ & $52.6(13.6)$ & $49.7(17.4)$ & 0.445 \\
\hline Sex (male/female) & $7 / 35$ & $12 / 23$ & $15 / 33$ & 0.162 \\
\hline Axial length (mm) & $23.3(0.6)$ & $23.5(1.1)$ & $23.9(1.7)$ & 0.053 \\
\hline Heart rate (beats/min) & $72.7(7.5)$ & $72.5(7.0)$ & $74.8(11.9)$ & 0.451 \\
\hline Systolic blood pressure (mmHg) & $126.2(8.0)$ & $127.2(12.0)$ & $127.1(10.5)$ & 0.892 \\
\hline Diastolic blood pressure (mmHg) & $73.2(6.3)$ & $76.5(6.7)$ & $75.1(6.1)$ & 0.071 \\
\hline Mean intraocular pressure $(\mathrm{mmHg})$ & $15.0(3.1)$ & $16.0(3.1)$ & $17.6(3.8)^{\mathrm{a}}$ & 0.002 \\
\hline
\end{tabular}

Values are presented as the mean (standard deviation).

aSignificant value compared to control ( $P<0.05, t$-test with Bonferroni correction).

Table 2 Ocular blood flow data of the three groups

\begin{tabular}{|c|c|c|c|c|}
\hline & Controls $(\mathrm{n}=42)$ & $A(G D)(\mathrm{n}=35)$ & $B(G O)(\mathrm{n}=48)$ & $P$ \\
\hline Pulse amplitude $(\mu \mathrm{l})$ & $3.5(0.9)$ & $3.2(0.9)$ & $2.3(0.8)^{\mathrm{a}}$ & $<0.001$ \\
\hline Pulse volume $(\mu \mathrm{l})$ & $8.7(2.4)$ & $7.6(2.4)$ & $4.9(1.9)^{\mathrm{a}}$ & $<0.001$ \\
\hline $\operatorname{POBF}(\mu \mathrm{l} / \mathrm{min})$ & $1255.4(295.1)$ & $1177.3(326.3)$ & $772.4(279.3)^{\mathrm{a}}$ & $<0.001$ \\
\hline
\end{tabular}

Values are presented as the mean (standard deviation).

a Significant value compared to control ( $P<0.05, t$-test with Bonferroni correction)

understood and may result from a complex interplay of genetic, environmental, and endogenous factors. ${ }^{19-21}$ At present, the development and severity of GO is unpredictable, and the treatment is still limited to symptom relief. Therefore, detection of its occurrence and activity in an early stage is important for the treatment strategy and prevention of GO.

Our study demonstrated that the PA, PV, and POBF in GD patients with ophthalmopathy were significantly lower than in those without ophthalmopathy and normal controls. However, there were no significant differences in these data between GD patients and the controls.

Apart from the effect of IOP, the disease status (GO) was still significantly correlated with the POBF. These results indicated that the total choroidal blood flow decreased with the development of ophthalmopathy in GD patients. Using colour Doppler imaging, Alp et $a l^{15}$ found increased velocity in the ophthalmic artery, central retinal artery, and central retinal vein and decreased velocity in the superior ophthalmic vein in patients with GO. ${ }^{15}$ The increased blood flow velocities in arteries possibly resulted either from the cardiovascular effect of hyperthyroidism or from orbital inflammation. ${ }^{15,16}$ However, the reduced POBF in GO patients in our study can be interpreted as the total effect of elevated venous pressure and increased resistance of choroidal vessels caused by elevated intraorbital pressure associated with severe autoimmune congestive inflammatory orbitopathy or contraction of the extraocular muscles against the fibrotic adhesions. Furthermore, it still needs more evidence to realize the role of the hypodynamic change in the pathogenesis of vision complication in GO patients.

In addition, our study also revealed that the mean IOP in patients with GO was significantly higher than that in normal controls and GD patients and it was also significantly correlated with the POBF. The prevalence of ocular hypertension has been reported to be higher in patients with GO. ${ }^{22-24}$ Since choroidal blood flow is ultimately derived from the same source as the optic nerve head (the short posterior ciliary arteries), the changes in choroidal blood flow may reflect the changes of blood flow in the optic nerve head. Therefore, a reduced ocular blood flow in GO patients, with a raised IOP, may be hazardous to patients by causing hypoperfusion or ischaemia of the optic nerve. This is especially so if the ocular circulation is already compromised such as in cases of glaucoma and ischaemic or compressive optic neuropathy. Early treatment including corticosteroids, radiation therapy, or surgical decompression is suggested in such patients.

In conclusion, POBF measurement can provide a reliable noninvasive method to detect haemodynamic change in GO patients. However, the clinical utility of ocular pulse analysis to differentiate clinical severity and treatment outcome of GO remains for future study.

\section{Acknowledgements}

This study was supported in part by a grant of National Science Council (No.NSC-91-2314-B-075-090). 


\section{References}

1 Silver DM, Farrell RA. Validity of pulsatile ocular blood flow measurements. Surv Ophthalmol 1994; 38 (Suppl): s72-s80.

2 Butt Z, O'Brien C. Reproducibility of pulsatile ocular blood flow measurements. J Glaucoma 1995; 4: 214-218.

3 Yang YC, Hulbert MF, Batterbury M, Clearkin LG. Pulsatile ocular blood flow measurements in healthy eyes: reproducibility and reference values. J Glaucoma 1997; 6: 175-179.

4 Silver DM, Farrell RA, Langham ME, O'Brien V, Schilder P. Estimation of pulsatile ocular blood flow from intraocular pressure. Acta Ophthalmol 1989; 191 (Suppl): 25-29.

5 Langham ME, Farrell RA, O'Brien V, Silver DM, Schilder P. Non-invasive measurements of pulsatile blood flow in the human eye. In: Lambrou GN, Greve EL (eds). Ocular Blood Flow in Glaucoma. Kugler and Ghedini: Amsterdam, 1989, pp. 93-99.

6 McKibbin M, Menage MJ. The effect of once-daily latanoprost on intraocular pressure and pulsatile ocular blood flow in normal tension glaucoma. Eye 1999; 13: 31-34.

7 Shaikh MH, Mars JS. The acute effect of pilocarpine on pulsatile ocular blood flow in ocular hypertension. Eye 2001; 15: 63-66.

8 Mori F, Konno S, Hikichi T, Yamaguchi Y, Ishiko S, Yoshida A. Pulsatile ocular blood flow study: decreases in exudative age related macular degeneration. $\mathrm{Br} J$ Ophthalmol 2001; 85: 531-533.

9 Chen SJ, Cheng CY, Lee AF, Lee FL, Chou JC, Hsu WM et al. Pulsatile ocular blood flow in asymmetric exudative age related macular degeneration. Br J Ophthalmol 2001; 85: 1411-1415.

10 Langham ME, Grebe R, Hopkins S, Marcus S, Sebag M. Choroidal blood flow in diabetic retinopathy. Exp Eye Res 1991; 52: 167-173.

11 Geyer O, Neudorfer M, Snir T, Goldstein M, Rock T, Silver DM et al. Pulsatile ocular blood flow in diabetic retinopathy. Acta Ophthalmol Scan 1999; 77: 522-525.
12 Yang YC, Kent D, Fenerty CH, Kosmin AS, Damato BE. Pulsatile ocular blood flow in eyes with untreated choroidal melanoma. Eye 1997; 11: 331-334.

13 Langham ME, Kramer T. Decreased choroidal blood flow associated with retinitis pigmentosa. Eye 1990; 4: 374-381.

14 Bahn RS, Heufelder AE. Pathogenesis of Graves' ophthalmopathy. N Engl J Med 1993; 329: 1468-1475.

15 Alp MN, Ozgen A, Can I, Cakar P, Gunalp I. Colour Doppler imaging of the orbital vasculature in Graves' disease with computed tomographic correlation. $\mathrm{Br} J$ Ophthalmol 2000; 84:

$$
\text { 1411-1415. }
$$

16 Kurioka Y, Inaba M, Kawagishi T, Emoto M, Kumeda Y, Inoue $\mathrm{Y}$, Morii $\mathrm{H}$, Nishizawa $Y$ et al. Increased retinal blood flow in patients with Graves' disease: influence of thyroid function and ophthalmopathy. Euro J Endocr 2001; 144: 99-107.

17 Bartley GB, Gorman CA. Diagnostic criteria for graves ophthalmopathy. Am J Ophthalmol 1995; 119: 792-795.

18 Wiersinga WM. Preventing Graves' ophthalmopathy. N Engl J Med 1998; 338: 121-122.

19 Jager MJ. Immunology and thyroid ophthalmopathy: where will the footprints lead us? Br J Ophthalmol 2000; 84: 446-447.

20 Weetman AP. Graves' disease. N Engl J Med 2000; 343: 1236-1248.

21 Prummel MF, Wiersinga WM. Smoking and risk of Graves' disease. JAMA 1993; 269: 479-482.

22 Cokerham KP, Pal C, Jani B, Wolter A, Kennerdell JS. The prevalence and implications of ocular hypertension and glaucoma in thyroid-associated orbitopathy. Ophthalmology 1997; 104: 914-917.

23 Ohtsuka K. Intraocular pressure and proptosis in 95 patients with Graves' ophthalmopathy. Am J Ophthalmol 1997; 124: 570-572.

24 Ohtsuka K, Nakamura Y. Open-angle glaucoma associated with Graves disease. Am J Ophthalmol 2000; 129: 613-617. 